\title{
Public Health and Medicine: Paradox or Complimentary?
}

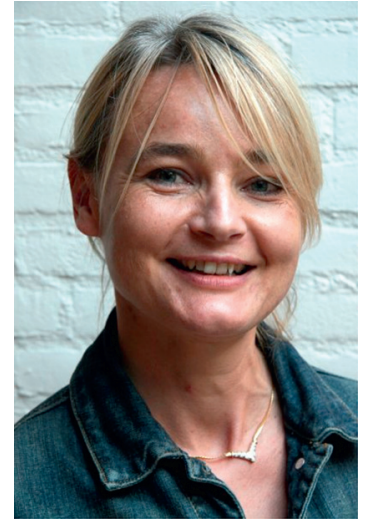

I warmly welcome you to this second issue of the International Journal of Medicine and Public Health. I feel that it is great that the need for Public Health: health promotion and disease prevention, is now being recognized within Medicine. However, at the same time it still is odd that medicine in itself does not imply public health. This is clearly illustrated by the addition of the word 'and' between these two important societal issues. The reason for public health and medicine not being integrated may originate from the focus on the individual from the medical point of view, whereas the focus of public health is on groups and society as a whole. So, medicine and public health are not one and the same, even though they strive the same goal: improving health. Nevertheless, improving health at a societal level, may differ from improving health at an individual level. This is where the 'Prevention paradox of Rose' comes in.

In order to improve health Geoffrey Rose (1926-1993), professor in epidemiology and preventive medicine, described two possible strategies: 'the high-risk strategy' and 'the population strategy', in his 1985 article "Sick Individuals and Sick Populations" and in his 1992 book "The Strategy of Preventive Medicine". The 'high-risk strategy' to prevention is a clinically-oriented approach targeting at individuals that can be described as 'being at risk' for the health threat concerned. The strength of this strategy is that it matches to the needs of the individual. Moreover, the health at an individual level will strongly improve. However, at the same time, the contribution to the overall population may be small. The 'population strategy' on the other hand is a public health-oriented approach, aiming at the entire population instead of at a small(-er) 'at risk group'. It starts with the recognition that the occurrence of common exposures and diseases reflects the functioning of society as a whole, improving health of the society as a whole. However, this strategy offers only a small benefit to each participating individual.

Although the initial idea of Rose was to make use both strategies at the same time: aiming at the entire population to improve health at a societal level, and aiming at high-risk groups to improve health at a individual level, his strategy became known as the 'prevention paradox', implying that one had to choose between strategies. This nicely reflects today's thinking of Medicine AND Public Health, whereas public health and it's nature of health promotion and disease prevention should be integrated into medicine. Let us hope that this journal's name can be changed within a couple of years into; The international Journal of public health medicine'...

I wish you lots of pleasure reading this very issue,

Anne Marie Plass Associate Editor IJMEDPH

DOI: 10.5530/ijmedph.2.2011.1 